Check for updates

Cite this: J. Mater. Chem. B, 2021, 9, 4535

Received 7th March 2021,

Accepted 2nd May 2021

DOI: $10.1039 / \mathrm{d} 1 \mathrm{tb} 00484 \mathrm{k}$

rsc.li/materials-b

\title{
Development of a 3D printable and highly stretchable ternary organic-inorganic nanocomposite hydrogel $\dagger$
}

\author{
Chen Hu, ${ }^{a}$ Malik Salman Haider, ${ }^{a}$ Lukas Hahn, ${ }^{a}$ Mengshi Yang ${ }^{a}$ and \\ Robert Luxenhofer (D) *ab
}

\begin{abstract}
Hydrogels that can be processed with additive manufacturing techniques and concomitantly possess favorable mechanical properties are interesting for many advanced applications. However, the development of novel ink materials with high intrinsic 3D printing performance has been proven to be a major challenge. Herein, a novel 3D printable organic-inorganic hybrid hydrogel is developed from three components, and characterized in detail in terms of rheological property, swelling behavior and composition. The nanocomposite hydrogel combines a thermoresponsive hydrogel with clay LAPONITE ${ }^{\circledR}$ XLG and in situ polymerized poly(N,N-dimethylacrylamide). Before in situ polymerization, the thermogelling and shear thinning properties of the thermoresponsive hydrogel provides a system well-suited for extrusion-based 3D printing. After chemical curing of the 3D-printed constructs by free radical polymerization, the resulting interpenetrating polymer network hydrogel shows excellent mechanical strength with a high stretchability to a tensile strain at break exceeding 550\%. Integrating with the advanced 3D-printing technique, the introduced material could be interesting for a wide range of applications including tissue engineering, drug delivery, soft robotics and additive manufacturing in general.
\end{abstract}

\section{Introduction}

Poly(2-oxazoline)s (POx) and poly(2-oxazine)s (POzi) are two diverse and closely related families of polymers that are readily accessible via living cationic ring opening polymerization and are highly tunable with respect to amphiphilic contrast and physico-chemical characteristics. ${ }^{1,2}$ They have been known for decades but have gained increasing interest from researchers

\footnotetext{
${ }^{a}$ Functional Polymer Materials, Chair for Advanced Materials Synthesis, Institute for Functional Materials and Biofabrication, Department of Chemistry and Pharmacy, Julius-Maximilians-University Würzburg, Röntgenring 11, 97070 Würzburg, Germany. E-mail: robert.luxenhofer@uni-wuerzburg.de, robert.luxenhofer@helsinki.fi

${ }^{b}$ Soft Matter Chemistry, Department of Chemistry, and Helsinki Institute of Sustainability Science, Faculty of Science, University of Helsinki, o0014 Helsinki, Finland

$\dagger$ Electronic supplementary information (ESI) available: Detailed description of POx- $b$-POzi diblock copolymer synthesis and characterization; optimization details of the hydrogel precursor solution composition in the view of rheology and mechanical strength; the thermogelling properties of POx-b-POzi/AA(or HEMA)/clay hydrogels; the hysteresis loops of the hydrogel at the maximum strains of $400 \%$ and $500 \%$; photographs of the hydrogel samples in consecutive cyclic tensile loading-unloading tests; the stability of POx-b-POzi/clay hydrogel in water; extrusion-based printing equipment; the long-term stability of 3D printed cube in $60^{\circ} \mathrm{C}$ silicone oil; 3D printed hydrogels for mechanical tests. (Fig. S1-S9) (PDF). Tensile test of the hydrogel (AVI). Printing of the hydrogel precursor (AVI). See DOI: $10.1039 / \mathrm{d} 1 \mathrm{tb} 00484 \mathrm{k}$
}

and industry in the last decade. ${ }^{2-7}$ One family member, poly(2ethyl-2-oxazoline) is approved as indirect food additive by the FDA and another member, a polymer-drug conjugate (SER-214) is currently in clinical trials. ${ }^{8}$ Along with the generally growing interest in POx and also POzi as an alternative to poly(ethylene glycol) (PEG) as gold standard for anti-fouling, non-toxic, nonimmunogenic and cyto-/biocompatible materials, POx and POzi are a fascinating and versatile platform for hydrogel preparation, especially for printable hydrogels, which can be integrated with the advanced additive manufacturing technique for different applications. ${ }^{9-12}$ In the past decade, a plethora of studies reported the design and development of several chemically crosslinked POx-based hydrogels, including the utilization of multifunctional 2-oxazoline monomers and macro-monomers with polymerizable groups, as well as the crosslinking of functional end- and side-groups with efficient coupling reactions. ${ }^{13-18}$ However, most of the reported approaches are not well-suited for additive manufacturing, and the hydrogels are typically brittle with limited stretchability. Especially for the most commonly used additive manufacturing or 3D printing technique of extrusionbased 3D printing, the hydrogels should meet certain criteria with respect to rheological properties, gelation mechanism and kinetics to ensure the desired printability and mechanical property. ${ }^{19-25}$ 
In addition to the many chemically crosslinked hydrogels, a few POx-based physical hydrogels have been described recently. ${ }^{26-30}$ Lorson et al. reported a thermogelling supramolecular hydrogel of a AB-type diblock copolymer comprising poly(2-methyl-2-oxazoline) (PMeOx) (A-block) and poly(2- $n$ propyl-2-oxazine) (PnPrOzi) (B-block) as a cytocompatible bioink for 3D bioprinting. This particular polymer selfassembles into polymer vesicles, which at higher concentration form a sponge-like structure leading to gelation. ${ }^{27}$ Very recently, Hahn et al. reported on a ABA-type triblock copolymer comprising again PMeOx as the hydrophilic block (A) and poly(2-phenyl-2-oxazine) as the hydrophobic block (B). This triblock copolymer showed an unique inverse thermogelation behavior with rather high storage moduli of up to $G^{\prime} \approx$ $110 \mathrm{kPa}^{29}$ Both synthetic thermogelling POx copolymers exhibit desirable rheological properties for 3D printing applications, in particular a beneficial combination of highly shear thinning character with rapid (re)gelation and sufficiently high yield stress needed for high-quality printing processes allowing good shape fidelity, which could be improved by the addition clay as a rheology modifier. ${ }^{20,31-33}$ However, while shear-thinning is an excellent feature for printing, the underlying weak physical crosslinks originating from hydrophobic interactions are not stable enough for subsequent practical applications, especially where an external mechanical load is required to withstand. The shear thinning impedes handling, overall performance and applicability. Therefore, to increase the spectrum of properties and potential application for POx-based hydrogel materials in the field of 3D printing, suitable additionally crosslinking methods are required to cure and stabilize the printed structures postfabrication.

Herein, we report a novel shear-thinning and thermogelling hydrogel system, PMeOx- $b$-PnPrOzi/poly( $N, N$-dimethylacrylamide)/clay (POx- $b$-POzi/PDMAA/clay), which is printable, and can be subsequently chemically cured by free radical polymerization to improve stability and elasticity. The developed POx-based hydrogel is studied with respect to printability and mechanical flexibility, and exhibited a comparable performance to the hydrogels prepared with traditional materials which have achieved significant achievements in tissue engineering and soft robotics but requiring additional support bath or rheology modifiers to realize the printability. ${ }^{34-36}$ In this system, the thermogelling POx- $b$-POzi copolymer acts as the first and rapidresponse physical network, which not only gives the hydrogel a satisfactory printability but also maintains the integrity of the printed structures temporarily. The second component PDMAA allows the formation of the interpenetrating network (IPN) hydrogel to improve significantly its long-term stability and mechanical properties. The third component, LAPONITE ${ }^{\mathbb{R}}$ XLG, is a commonly used synthetic hectorite clay for preparing high-performance nanocomposite hydrogels, and should add strength to the final IPN and concurrently improve the printability. ${ }^{33,37-39}$ Finally, a variety of 3D constructs were printed successfully with good shape fidelity and interesting mechanical properties, demonstrating a proof-ofconcept for the use of this novel material.

\section{Materials and methods}

\section{Materials}

All substances and reagents were obtained from Sigma-Aldrich (Steinheim, Germany) or Acros (Geel, Belgium) and were used as received unless otherwise stated. Reagents used for synthesis of POx- $b$-POzi diblock copolymer, specifically the initiator methyl trifluoromethylsulfonate (MeOTf), the monomer 2methyl-2-oxazoline (MeOx), and solvent benzonitrile (PhCN) were dried by refluxing over $\mathrm{CaH}_{2}$ under dry argon atmosphere followed by subsequent distillation. The second monomer 2-n-propyl-2-oxazine (nPrOzi), was synthesized by an adapted standard procedure, ${ }^{40}$ distilled over $\mathrm{CaH}_{2}$ and stored under dry argon. LAPONITE ${ }^{\circledR}$ XLG $\left(\left[\mathrm{Mg}_{5.34} \mathrm{Li}_{0.66} \mathrm{Si}_{8} \mathrm{O}_{20}(\mathrm{OH})_{4}\right] \mathrm{Na}_{0.66}\right)$ was purchased from BYK-Chemical GmbH (Wesel, Germany) and used as received. Deionized (DI) water was used throughout the experiments.

\section{Synthesis and characterization of POx-b-POzi diblock copolymer}

The POx- $b$-POzi diblock copolymer was synthesized using MeOTf to initiate a cationic ring-opening polymerization of monomers MeOx followed by nPrOzi, as previously described.,27 Specifically, under dry and inert conditions, $0.48 \mathrm{~g}$ (2.92 mmol, 1 equiv.) MeOTf and $24.92 \mathrm{~g}$ (292.8 mmol, 100 eq.) of MeOx were added to $56.58 \mathrm{~mL}$ of dry benzonitrile (PhCN) in a dried flask and polymerized at $120{ }^{\circ} \mathrm{C}$ for $4 \mathrm{~h}$. Full monomer conversion was verified by ${ }^{1} \mathrm{H}$ NMR before addition of the monomer for the second block. After the reaction mixture cooled to ambient temperature, $37.27 \mathrm{~g}$ nPrOzi (293.0 mmol, 100 eq.) dissolved in $70.02 \mathrm{~mL}$ of dry PhCN was added, under continuous stirring at $140{ }^{\circ} \mathrm{C}$ overnight. Subsequently, the mixture was cooled again to room temperature, and $1.63 \mathrm{~g}$ (8.76 mmol, 3 eq.) of 1-Boc-piperazine was added. The reaction mixture was stirred overnight at $40{ }^{\circ} \mathrm{C}$. After cooling to room temperature, $0.40 \mathrm{~g}$ potassium carbonate $(2.92 \mathrm{mmol}$, 1 eq.) was added and the mixture was again stirred for $5 \mathrm{~h}$. Thereafter, the solvent was removed under reduced pressure, the residual was dissolved in deionized water, and dialyzed against deionized water using a dialysis membrane with a molecular weight cut-off (MWCO) of $10 \mathrm{kDa}$ for 2 days. The product was obtained after lyophilization, and named shortly as POx- $b$-POzi.

The chemical structure of POx- $b$-POzi was confirmed by ${ }^{1} \mathrm{H}$ NMR on Fourier $300(300.12 \mathrm{MHz}$ ) Bruker BioSpin (Rheinstetten, Germany) at $298 \mathrm{~K}$ with $\mathrm{CDCl}_{3}$ as solvent. The data was further analyzed by MestReNova software. Gel permeation chromatography (GPC) was performed on an Agilent 1260 Infinity, System Polymer Standards Service (Mainz, Germany) with hexafluoroisopropanol (HFIP) containing $3 \mathrm{~g} \mathrm{~L}^{-1}$ potassium trifluoroacetate (KTFA) as eluent; precolumn: $50 \mathrm{~mm} \times 8 \mathrm{~mm}$ PSS PFG linear M; 2 columns: $300 \mathrm{~mm} \times 8 \mathrm{~mm}$ PSS PFG linear M (particle size $7 \mu \mathrm{m}$; pore size $0.1-1000 \mathrm{kDa}$ ). The columns were kept at $40{ }^{\circ} \mathrm{C}$ and flow rate was $0.7 \mathrm{~mL} \mathrm{~min}^{-1}$. Prior to GPC measurement, sample was filtered through a $0.2 \mu \mathrm{m}$ Teflon filter (Thermo Scientific) to remove particles, if any. 


\section{POx- $b$-POzi/PDMAA/clay hydrogels preparation}

The POx- $b$-POzi/PDMAA/clay nanocomposite hydrogels were prepared by thermogelation and free radical polymerization from the initial solutions consisting of clay, POx- $b$-POzi, DMAA, $N, N^{\prime}$-methylenebisacrylamide (MBAA), and initiator potassium persulfate (KPS). Exemplarily, the preparation of POx- $b$-POzi/ PDMAA/clay hydrogel was performed as follows where the mass fraction of POx- $b$-POzi, PDMAA and clay were 30, 10 and $1.2 \mathrm{wt} \%$. First, $2 \mathrm{wt} \%$ clay aqueous stock solution was prepared by dispersing the accurate amount of dry LAPONITE ${ }^{\circledR}$ XLG powder in deionized water with continuous stirring to ensure the thorough hydration of LAPONITE $^{\circledR}$ powder, until a homogeneous and transparent solution was obtained (at least $24 \mathrm{~h}$ ). Second, $0.4 \mathrm{~g}$ DMAA and $1.2 \mathrm{~g}$ lyophilized POx- $b$-POzi copolymer were added into $2.4 \mathrm{~g} \quad 2 \mathrm{wt} \%$ clay solution. Thereafter, the mixture was stored at $7{ }^{\circ} \mathrm{C}$ overnight to allow complete dissolution followed by the addition of $0.002 \mathrm{~g}$ MBAA and $0.002 \mathrm{~g}$ initiator KPS, which are both at $0.5 \mathrm{wt} \%$ relative to the weight of DMAA, to obtain a homogeneous dispersion. Subsequently, the dispersion was transferred into a reaction cell consisting of two polymethyl methacrylate (PMMA) plates separated by a polyvinyl chloride (PVC) spacer to produce a physical cross-linked POx- $b$-POzi/DMAA/clay hydrogel at room temperature. Third, the mold was placed in an oven at $60{ }^{\circ} \mathrm{C}$ for $6 \mathrm{~h}$ to initiate the free radical polymerization of DMAA. The developed POx- $b$-POzi/PDMAA/clay hydrogels were obtained after taken out of the mould. For comparison, the hydrogel POx- $b$-POzi/PDMAA without clay was also prepared using DI water to replace $2 \mathrm{wt} \%$ clay aqueous solution as solvent.

\section{Rheological measurements}

Rheological measurements were performed using the MCR 301 rheometer from Anton Paar (Ostfildern, Germany) employing a $25 \mathrm{~mm}$ diameter parallel-plate geometry. A Peltier system was employed for temperature control. First, under a constant angular frequency and strain of $10 \mathrm{rad} \mathrm{s}^{-1}$ and $0.5 \%$ respectively, the temperature sweeps from 5 to $45{ }^{\circ} \mathrm{C}$ were carried out at a heating rate of $0.05{ }^{\circ} \mathrm{C} \mathrm{s}^{-1}$ to study the thermogelling behavior. Second, amplitude sweeps in the oscillation strain range of 0.01$500 \%$ was performed at a constant angular frequency of $10 \mathrm{rad} \mathrm{s}^{-1}$, from which the linear viscoelastic (LVE) range was determined. Third, the frequency sweep was performed in an angular frequency range of $0.1-100 \mathrm{rad} \mathrm{s}^{-1}$ at a certain strain of $0.1 \%$ (within the LVE range obtained from the amplitude sweep). Finally, the steady-state shear flow from 0.01 to $1000 \mathrm{~s}^{-1}$ of shear rate were performed to characterize the shear thinning behavior. Except for the temperature sweep, all tests were performed at $37^{\circ} \mathrm{C}$. An aqueous solvent trap was utilized in all experiments to mitigate drying effects.

\section{Mechanical tests}

The mechanical performance of the hydrogel was tested by universal testing machine Zwick Roell Z010 (Ulm, Germany). Force sensors of $100 \mathrm{~N}$ for tensile test and $10 \mathrm{kN}$ for compressive test were applied at room temperature. In tensile tests, the hydrogel was cut into long stripes with a length of $35 \mathrm{~mm}$ and a cross-sectional area of $5 \times 1 \mathrm{~mm}$. The original gauge length between the clamps of every samples was recorded followed by test performed at a constant velocity of $100 \mathrm{~mm} \mathrm{~min}^{-1}$. The nominal stress was determined as the loading force divided by the original specimen cross-sectional area, the nominal strain was defined as the deformed length divided by the original gauge length, and the Young's modulus were calculated from the slope of the linear region in the $0-20 \%$ strain range of the stress-strain curves. In hysteresis measurements, the hydrogel was initially loaded to a predetermined strain and then unloaded to zero force at a constant velocity of $100 \mathrm{~mm} \mathrm{~min}^{-1}$. Such cyclic loading-unloading tensile tests were programmed to repeat 10 times. Additionally, the cylinder-shape hydrogel with a height of $7 \mathrm{~mm}$ and a diameter of $12 \mathrm{~mm}$ was used for compression tests at a speed of $0.7 \mathrm{~mm} \mathrm{~min}^{-1}$. For all tests, the samples surface was greased with olive oil to prevent water evaporation during testing.

\section{Hydrogel characterizations}

Swelling ratio (SR). The SR of the hydrogel was assessed using a gravimetric method. Initially, the dry weight $\left(W_{\mathrm{d}}\right)$ of hydrogels was noted, followed by immersion in DI water at $37{ }^{\circ} \mathrm{C}$. At predefined time points, the wet hydrogels were taken, the excess surface liquid was cleared using blotting paper, and the swelled weight $\left(W_{t}\right)$ was recorded. The study was conducted under identical conditions in triplicates, and the SR was calculated using the equation $\mathrm{SR}=\left(W_{t}-W_{\mathrm{d}}\right) / W_{\mathrm{d}}$.

Sol content. Sol content of the hydrogel was calculated using formula $\left(M_{0}-M_{1}\right) / M_{0}(n=3)$, where $M_{0}$ is the dry mass of the as-prepared chemically crosslinked polymer hybrid and $M_{1}$ is the final mass of lyophilized gel after allowing it to swell for three days in DI water at $37{ }^{\circ} \mathrm{C}$ with the water replaced every $12 \mathrm{~h}$.

Differential scanning calorimetry (DSC). DSC measurements were performed on DSC 204 F1 Phoenix equipped with a CC200 F1 controller from Netzsch (Selb, Germany). The dynamic scans were recorded in nitrogen atmosphere with the heating rate of $10 \mathrm{~K} \mathrm{~min}^{-1}\left(25-240{ }^{\circ} \mathrm{C}\right)$ and subsequently cooled to $-50{ }^{\circ} \mathrm{C}$. The samples were heated and cooled two additional times from -50 to $200{ }^{\circ} \mathrm{C}$ (three heating and two cooling cycles). For DSC studies, samples $(\sim 5 \mathrm{mg})$ were placed into flat bottom aluminum pans with crimped-on lids (pierced on the top).

3D printing. Printing experiments were performed using the extrusion-based 3D bioprinter BIO X from CELLINK (Gothenburg, Sweden) equipped with pneumatic driven print head and a $0.33 \mathrm{~mm}$ inner diameter precision needle $(23 \mathrm{G})$. The extrusion pressure was controlled and varied according to different userdefined printing structures which are programmed by G-code. The prepared POx- $b$-POzi/DMAA/clay hydrogel was loaded as the ink into a printing cartridge in liquid (cooled) form conveniently because of its thermogelling property, and then kept in $7{ }^{\circ} \mathrm{C}$ fridge before printing to eliminate bubbles. To avoid the dehydration effect and the inhibition on DMAA polymerization by oxygen, silicone oil which was purged with argon gas for few hours was used as protective bath, and kept at $60{ }^{\circ} \mathrm{C}$ prior to use. 
The POx- $b$-POzi/DMAA/clay-loaded printing cartridge was mounted in the 3D printer immediately after removal from the fridge, and driven pneumatically through the nozzle to print the hydrogel on a Petri dish placed on $37{ }^{\circ} \mathrm{C}$ print-bed. The printing speed was fixed at $5 \mathrm{~mm} \mathrm{~s}^{-1}$ in all printing experiments. Immediately after printing, the printed structures were chemically cured by transferring the Petri dish to the $60{ }^{\circ} \mathrm{C}$ oven for $6 \mathrm{~h}$ for DMAA polymerization, wherein the pre-heated $60{ }^{\circ} \mathrm{C}$ argonpurified silicone oil was used as protective bath. Thereafter, the cured 3D structures of POx- $b$-POzi/PDMAA/clay hydrogel were obtained after removing the silicone oil bath.

\section{Results and discussion}

\section{POx-b-POzi polymer synthesis and characterizations}

The diblock copolymer POx- $b$-POzi was synthesized by sequential cationic ring opening polymerization of MeOx and nPrOzi as previously reported and characterized. ${ }^{1,27}$ From the signals in the ${ }^{1} \mathrm{H}$ NMR spectrum with chemical shifts at $2.09 \mathrm{ppm}\left(\mathrm{CH}_{3}\right.$ of PMeOx) and $0.93 \mathrm{ppm}\left(\mathrm{CH}_{3}\right.$ of PnPrOzi), as well as the GPC analysis (Fig. 1 and Fig. S1, ESI $\dagger$ ), the relative block lengths and diblock copolymer composition was determined to be $\mathrm{PMeOx}_{110^{-}}$ $b$-PnPrOzi ${ }_{109}$ with a number-average molar mass $\left(M_{\mathrm{n}}\right)$ of around $23 \mathrm{~kg} \mathrm{~mol}{ }^{-1}$ and a dispersity of $D=1.3$, indicating that the desired diblock copolymer was obtained with reasonable control and definition. We choose the degree of polymerization of around 100 for both blocks as this yields a hydrogel with a $T_{\text {gel }}$ at slightly below room temperature, which we deemed favorable for this proof-of-concept. Short block lengths will lead to higher $T_{\text {gel. }}{ }^{27}$

\section{POx-b-POzi/PDMAA/clay hydrogel preparation}

The ternary POx- $b$-POzi/PDMAA/clay hydrogel was prepared using a two-step approach (Fig. 2a). First, the components were mixed in the desired ratios in the cold and allowed to undergo the thermogelation by allowing to warm to room temperature. Then, DMAA was polymerized using free radical polymerization initiated by KPS. An initial screening of the composition of the ternary hydrogel precursor mixtures with respect to their rheological properties was used to determine their viscoelastic properties in order to estimate printability and elastic properties after curing (see Tables S1, S2 and Fig. S2, and Discussion in ESI $\dagger$ ). The composition of $1.2 \mathrm{wt} \%$ clay, $30 \mathrm{wt} \%$ POx- $b$-POzi and $10 \mathrm{wt} \%$ DMAA with an addition of crosslinker MBAA and initiator KPS (both $0.5 \mathrm{wt} \%$ relative DMAA) in DI water was chosen for further experiments.

In the first step, the homogeneous POx- $b$-POzi/DMAA/clay mixture (with MBAA/KPS) was transferred into a mold and subsequently allowed to complete the thermogelation at room temperature. The thermoresponsive diblock copolymer POx- $b$ POzi self-assembles into spherical vesicles, and above a critical temperature, a sponge-like network leading the macroscopic physical gelation was observed. ${ }^{27}$ We have recently also studied the binary POx- $b$-POzi/clay system and found that the inorganic additive does not negatively affect this thermogelation. ${ }^{33}$ In a second step, the DMAA was polymerized at $60{ }^{\circ} \mathrm{C}$ for $6 \mathrm{~h}$ to produce the POx- $b$-POzi/PDMAA/clay IPN nanocomposite hydrogel. After the chemical curing step of DMAA, the ternary hybrid hydrogel lost its reversible thermogelation property, and transformed from a transparent to white/opaque material (Fig. 2b). Most interestingly, we noticed that the obtained POx- $b$-POzi/PDMAA/clay hydrogels show a very elastic behavior, can sustain high stretching deformations and recover quickly without any observable damage (Fig. 2c).

We also investigated acrylic acid (AA) and 2-hydroxyethylmethacrylate (HEMA) (Fig. S3, ESI $\dagger$ ) as alternative hydrogel precursors. However, the addition of these two monomers affected the thermogelation of POx- $b$-POzi in an unfavorable manner. All investigated compositions remained as gel at all

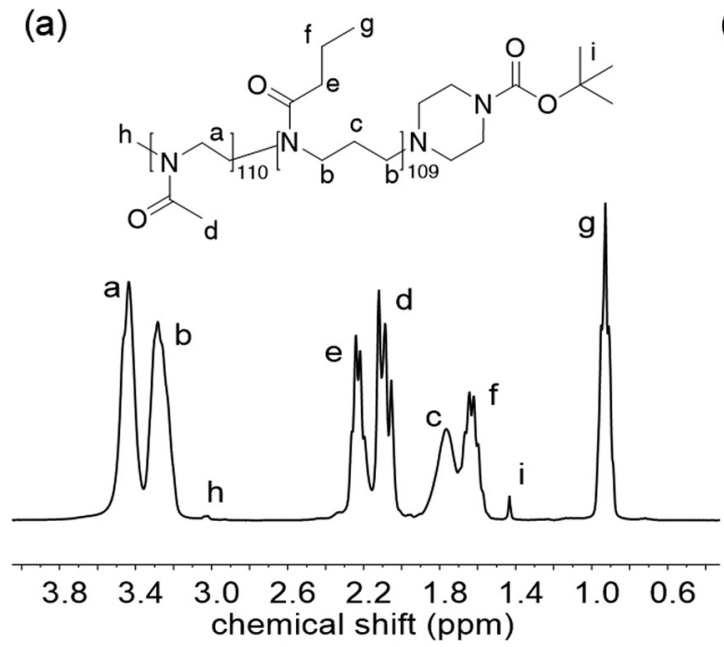

(b)

\begin{tabular}{|c|c|}
\hline $\begin{array}{c}\text { Polymer } \\
\text { composition }\end{array}$ & $\mathrm{PMeOx}_{110^{-} b-\mathrm{PnPrOzi}}{ }_{109}$ \\
\hline$M_{n}^{a}$ & 23 \\
\hline$M_{n}^{b}$ & 7.3 \\
\hline$M_{w}^{b}$ & 9.7 \\
\hline $\boldsymbol{D}^{\mathrm{b}}$ & 1.3 \\
\hline
\end{tabular}

standards.

Fig. 1 (a) Chemical structure of the synthesized diblock copolymer POx-b-POzi and the corresponding ${ }^{1} \mathrm{H}$ NMR spectrum (CDCl $; 300 \mathrm{MHz} ; 298 \mathrm{~K}$ ). (b) Polymer composition, molar masses $\left(\mathrm{kg} \mathrm{mol}^{-1}\right.$ ), and dispersity of the synthesized POx-b-POzi diblock copolymer obtained via ${ }^{1} \mathrm{H}$ NMR and GPC with HFIP as eluent 
(a)

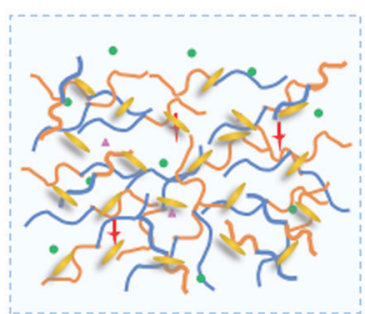

POx-b-POzi/DMAA/clay sol

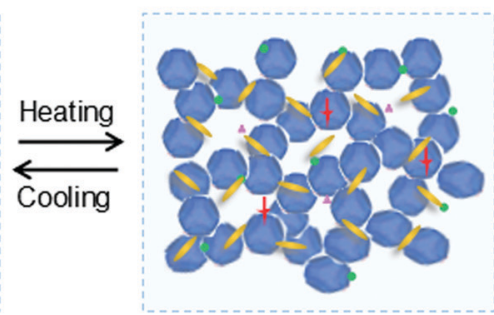

POx-b-POzi/DMAA/clay gel

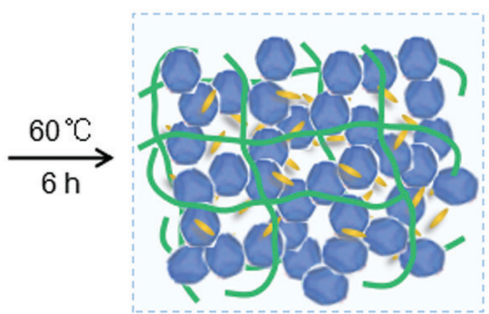

POx-b-POzi/PDMAAclay hydrogel i)

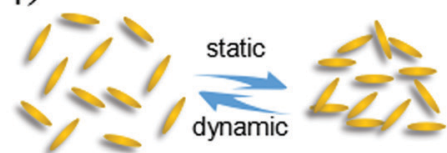

clay ii)

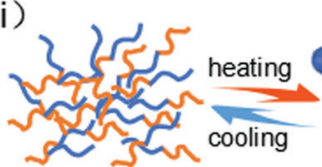

POx-b-POzi

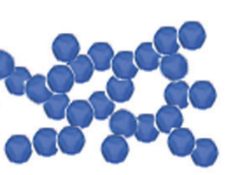

(c)

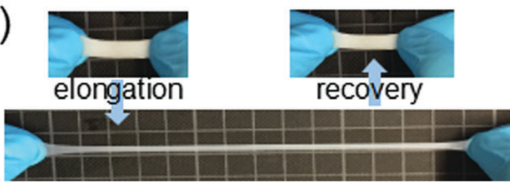

Fig. 2 (a) Schematic illustration for the preparation of POx-b-POzi/PDMAA/clay hydrogel via the strategy of thermogelation and subsequent free radical polymerization from three components: (i) clay LAPONITE ${ }^{\mathbb{R}}$ XLG, (ii) thermoresponsive diblock copolymer POx-b-POzi and (iii) chemically crosslinked PDMAA network. (b) Photographs indicating the thermoreversible sol-gel transition of POx-b-POzi/DMAA/clay mixture and the state after curing. (c) Photographs demonstrating the high stretchability and recovery of POx-b-POzi/PDMAA/clay hydrogel.

investigated temperatures. We hypothesize that these monomers compete with the water molecules more effectively, preventing hydration of the nPrOzi block at lower temperatures and therefore, liquefaction of the gels.
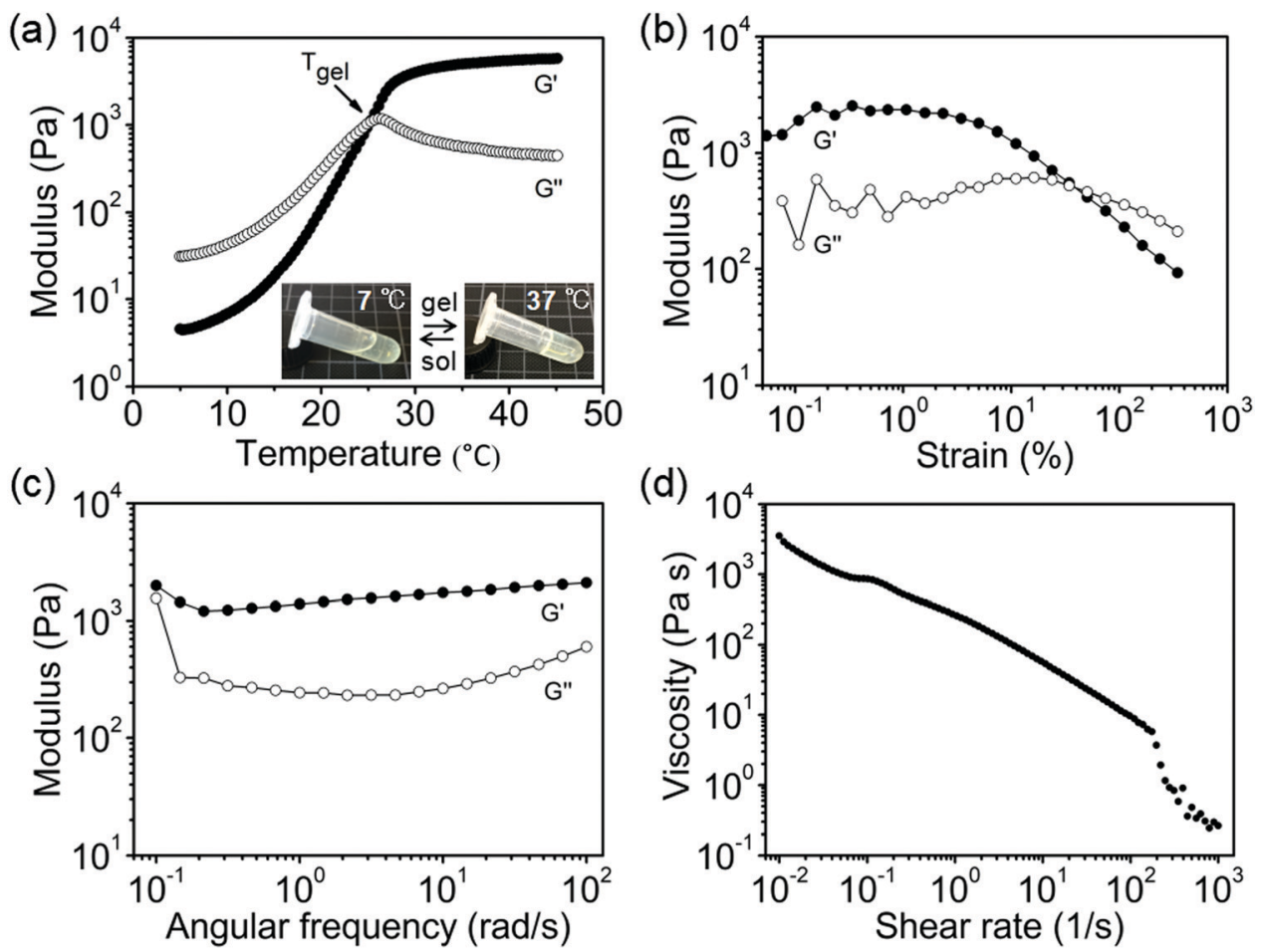

Fig. 3 Rheological characterizations of POx-b-POzi/DMAA/clay hydrogel. (a) A temperature sweep yields the gelation temperature at the intersection of $G^{\prime}$ and $G^{\prime \prime}$. Inset: Pictures visualizing the sol-gel transition. (b) Amplitude sweep to determine the LVE range. (c) Frequency sweep for the hydrogel under an applied strain of $0.1 \%$. (d) Viscosity vs. shear rate, demonstrating the shear-thinning behavior of the hydrogel. 


\section{Rheological properties of POx-b-POzi/DMAA/clay precursor}

Rheological measurements were carried out to understand the viscoelastic behavior and printability of POx-b-POzi/DMAA/clay system (with MBAA and initiator KPS) prior to chemical crosslinking. Initially, the thermogelling behavior was investigated by a dynamic oscillation temperature sweep. The intersection of storage modulus $\left(G^{\prime}\right)$ and loss modulus $\left(G^{\prime \prime}\right)$ defines the gel point, and the corresponding temperature as gel temperature ( $\left.T_{\text {gel }}\right)$ (Fig. 3a). The POx- $b$-POzi/DMAA/clay mixture is liquid at low temperatures $\left(G^{\prime \prime}>G^{\prime}\right)$. Upon heating, $G^{\prime}$ and $G^{\prime \prime}$ increase with increasing temperature. At around $25{ }^{\circ} \mathrm{C}\left(T_{\text {gel }}\right), G^{\prime} \geq G^{\prime \prime}$, before $G^{\prime}$ of the hybrid gel finally reaches a plateau at $G^{\prime} \sim$ $6 \mathrm{kPa}$. The slightly lower $T_{\text {gel }}$ and higher $G^{\prime}$ found here compared to the value of POx- $b$-POzi/DMAA/clay mixture without MBAA and KPS (Fig. S2, ESI $\dagger$ ) may be attributed to partial polymerization of DMAA in the course of the experiment.

Subsequently, an amplitude sweep was conducted to assess the linear viscoelastic region (LVE) range, which was determined at the onset of the decrease of $G^{\prime}$ (Fig. 3b). The subsequent dynamic frequency sweep (strain $=0.1 \%$, within the LVE range) showed a dominating $G^{\prime}$ value over $G^{\prime \prime}$ in the entire investigated frequency range (0.1-100 $\left.\mathrm{rad} \mathrm{s}^{-1}\right)$, indicating its stable gel-like character (Fig. 3c). Moreover, increasing the shear rate resulted in decrease of the hydrogel's viscosity, confirming that the POx-b-POzi/DMAA/clay system remains a material with pronounced shear-thinning character (Fig. 3d).
Among others, thermogelling and shear-thinning are very valuable properties for extrusion-based 3D printing, which facilitate the smooth printing process as well as the 3D structure maintenance after printing.

\section{Mechanical properties of POx-b-POzi/PDMAA/clay hydrogel}

Besides the investigation of the hydrogel precursors rheological properties for printability assessment, the mechanical properties of the hydrogel after chemical curing were studied. We found that the materials became highly stretchable after curing as shown in tensile tests (Fig. 4a and Video S1, ESI †). The elongation at break was a remarkable $550 \%$ with a Young's modulus and breaking strength of $12 \mathrm{kPa}$ and $25 \mathrm{kPa}$, respectively (Fig. 4b). We believe this report is the first report showing such highly stretchable hydrogels based on POx, although generally speaking, it is well known that nanocomposite hybrid hydrogels, in particular in combination with an IPN (here with PDMAA) often exhibit remarkable mechanical properties, such as good recovery, excellent flexibility, and stretchability. Even though the elongation at break was reasonably high in the absence of clay, its incorporation significantly improved it (Fig. S2, ESI $\dagger$ ). Cyclic loading-unloading tests with various maximum strains of $100 \%$, $200 \%$ and $300 \%$ were also performed to evaluate the energy dissipation capacity of the hydrogels (Fig. 4b). Apparent hysteresis loops, indicating the energy dissipation, were observed in the loading-unloading cycle of hydrogels, suggesting that there are non-covalent interactions in the nanocomposite hydrogels which (a)
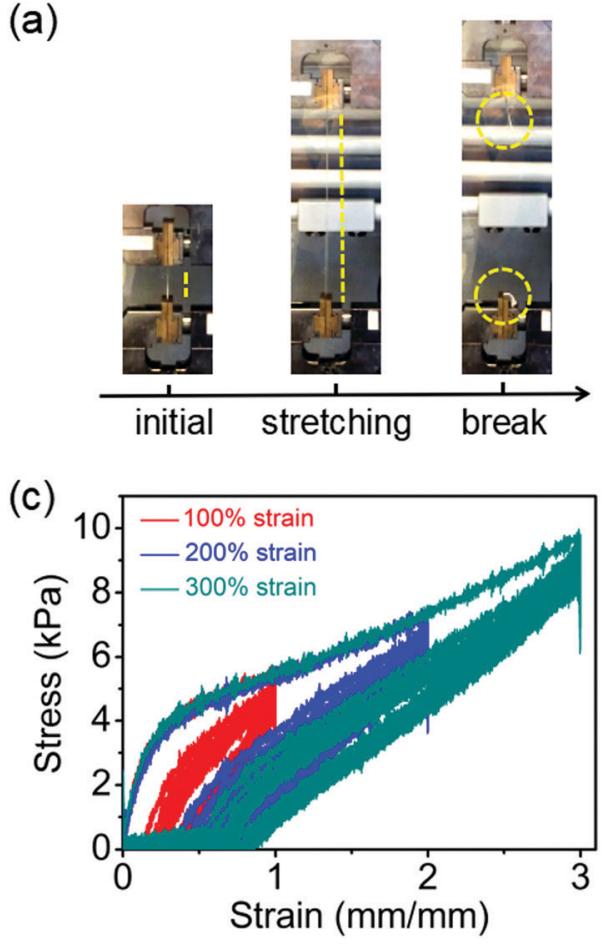

(b)

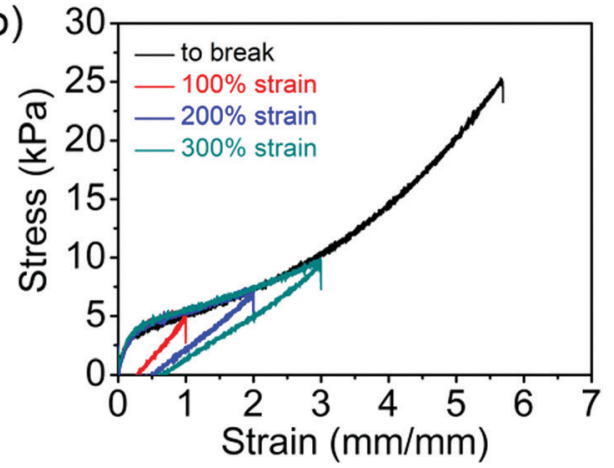

(d)

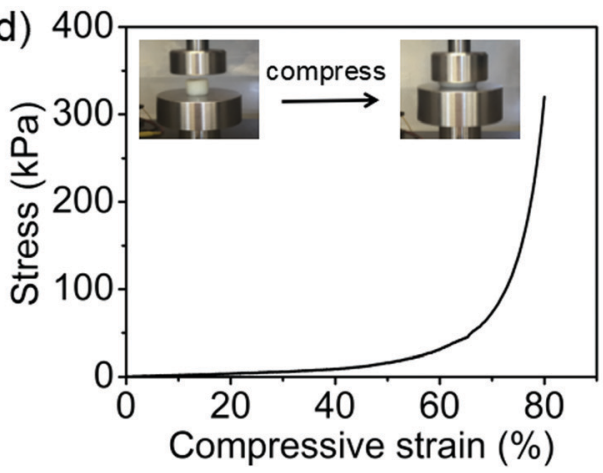

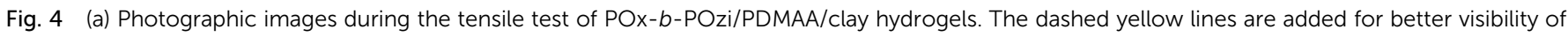

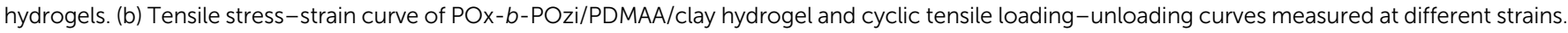

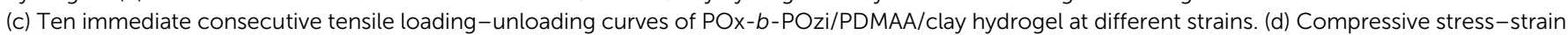

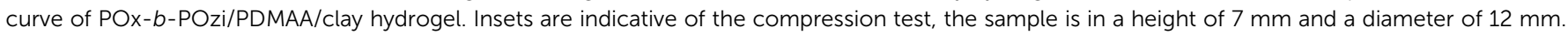


can dissipate energy effectively, and thus resulting in the hysteresis effect as well as facilitating the hydrogel's stretchability. After removing the tensile force from the hydrogels, the strains returned with a small hysteresis below $50 \%$. This shows that the hydrogels have a strong elastic nature but also that some plastic deformation occurs.

Additionally, consecutive cyclic tensile loading-unloading tests were also performed to investigate the fatigue resistance (Fig. 4c). Generally, during the ten immediate consecutive loading-unloading cycles, the hysteresis loops remarkably decreased, in particular after the first loading. Upon subsequent loading, an inverse relationship was observed between hysteresis loop and residual strain (gradual decrease of hysteresis loop area with the gradual increase of residual strain) throughout the entire loading-unloading process. Important to note, the tensile stress also decreased in the successive cycles, without appearance of any visible cracks or fractures.

These results suggest that, the energy dissipation is due to the destruction of internal structures of the gel. In addition, loading-unloading cycles performed at higher critical strains of $400 \%$ and $500 \%$ showed a qualitatively similar picture as with lower strains (Fig. S4, ESI $\dagger$ ). When allowing the strained hydrogels to recover for $2 \mathrm{~h}$ at room temperature after ten cyclic loading-unloading tests, the test specimen recovered their original dimensions to some extent (Fig. S5, ESI $\dagger$ ). Apart from the tensile stress-strain, a uniaxial compressive test was carried out to assess the compressive strength of the hybrid IPN hydrogel (Fig. 4d). A compressive modulus of $0.16 \pm 0.01 \mathrm{kPa}$ was determined, and a compressive strength of $320 \mathrm{kPa}$ at $80 \%$ deformation was measured without the material collapsing. It should be noted that these mechanical tests were conducted at room temperature without specific temperature control. While the PDMAA network is chemically crosslinked, there remains a thermoresponsive component from POx- $b$-POzi in the IPN. In future work, it will be interesting to study the changes in mechanical properties of the ternary IPN with the temperature. Such detailed analysis, however, is outside the scope of the present contribution.

\section{Characterizations of POx-b-POzi/PDMAA/clay hydrogel}

The swelling behavior of the novel ternary hybrid materials was investigated at $37{ }^{\circ} \mathrm{C}$ in DI water (Fig. 5a). The hydrogels showed rapid swelling within the first $6 \mathrm{~h}$. After $24 \mathrm{~h}$, the swelling degree leveled off with only minor increase up to 4 days. The swelling ratio after 4 days was found to be $1100 \%$, which is in the expected range for non-ionic hydrogels of POx and PDMAA. ${ }^{15,18,41}$ At the same time, the sol content of the hydrogels was measured as around $39.94 \pm 0.05 \%$ with a corresponding gel content of $60.06 \%$. Considering that the original solid content of PDMAA/clay in the dry hybrid gels makes up about $27.18 \%$ (POx- $b$-POzi is around $72.82 \%$ ), it is clear that a considerable amount of POx- $b$-POzi remained in the hydrogel despite extensive washing. This could be due to two main reasons, i.e., that polymer is very effectively physically trapped and/or in part covalently linked to the PDMAA network by virtue of chain transfer reactions in the free radical polymerization.

Additionally, DSC analysis was carried out on the dried hydrogels before (POx- $b$-POzi/DMAA/clay) and after chemical crosslinking (POx- $b$-POzi/PDMAA/clay), as well as after solextraction (POx-b-POzi/PDMAA/clay extr.) (Fig. 5b). The merely physically crosslinked hydrogel POx- $b$-POzi/DMAA/clay has two glass transition temperatures $\left(T_{\mathrm{g}}\right)$ at $8.8{ }^{\circ} \mathrm{C}$ and $78.8{ }^{\circ} \mathrm{C}$ corresponding to the two blocks of POx- $b$-POzi (Fig. $5 \mathrm{~b}$, black trace). ${ }^{33}$ In contrast, the chemically crosslinked POx- $b$-POzi/PDMAA/clay hydrogels showed three $T_{\mathrm{g}}$ values before and after extraction (Fig. 5b, red and blue traces). The new $T_{\mathrm{g}}$ around $127{ }^{\circ} \mathrm{C}$ corresponds to the newly formed PDMAA. ${ }^{41}$ The appearance of three individual $T_{\mathrm{g}}$ values in the crosslinked interpenetrating network, especially after extraction, confirms the presence of POx- $b$-POzi after extensive extraction. Important to note, without the PDMAA, the POx- $b$-POzi/clay hydrogels dissolve rapidly in water (Fig. S6, ESI $\dagger$ ). The presence of three $T_{\mathrm{g}}$ values also points towards microphase separation of all three polymers. The slight increase in $T_{\mathrm{g}}$ of the POx- $b$-POzi copolymer may be attributed to chain transfer reactions, covalently linking the diblock copolymer to the PDMAA and reducing their chains segment mobility.

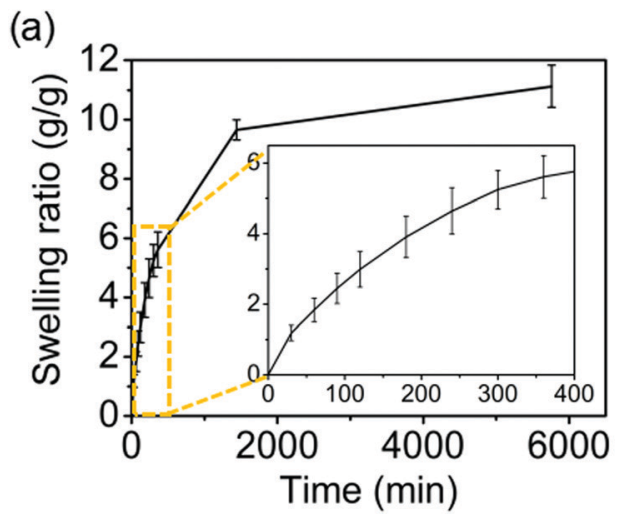

(b)

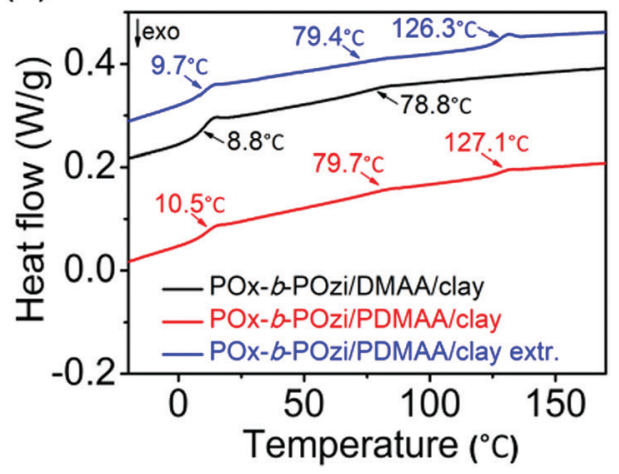

Fig. 5 (a) Swelling behavior of POx-b-POzi/PDMAA/clay hydrogel. (b) DSC analysis of the dried hydrogels POx-b-POzi/DMAA/clay before chemical crosslinking, POx-b-POzi/PDMAA/clay after chemical crosslinking, and POx-b-POzi/PDMAA/clay extr. after sol-extraction. 


\section{D printing}

The POx- $b$-POzi/DMAA/clay hydrogel was printed by an extrusion-based 3D printer to investigate their 3D printability (Fig. S7, ESI $\dagger$ ). A 20-layer hollow five-pointed star $(7.28 \times 7.28 \times$ $5 \mathrm{~mm})$ and a 20-layered cube $(10 \times 10 \times 5 \mathrm{~mm})$ were printed using extrusion pressure of $190 \mathrm{kPa}$ and $155 \mathrm{kPa}$, respectively (Fig. 6). These pressures were established empirically in preliminary test prints. The POx- $b$-POzi/DMAA/clay physical hydrogel could be printed smoothly through a relatively fine needle $(23 \mathrm{G})$, and recovered to solid-like gel state immediately after extrusion onto the $37{ }^{\circ} \mathrm{C}$ print bed. The combination of the highly shear thinning character and rapid thermogelling, allows avoidance of extra crosslinking agents at this point and facilitates the high-quality printing process without undesired fusion and collapse of the printed structures (Video S2, ESI $\dagger$ ). The designed 3D constructs of a cube and a five-pointed star were printed successfully with high shape fidelity, demonstrating that the POx- $b$-POzi/DMAA/clay hydrogel are an excellent ink for the fabrication of $3 \mathrm{D}$ constructs, that can support many times their own weight as evidenced by the excellent stackability during printing. Unsurprisingly, the printed structures without chemical curing collapsed gradually when the temperature drops below the $T_{\text {gel }}$, again confirming the reversible thermogelling property of the POx-b-POzi/DMAA/clay hydrogel (Fig. 6b, right side). In contrast, the printed constructs retained their structural integrity for a long time when the temperature was kept above $T_{\text {gel }}$, for example when incubation at $60{ }^{\circ} \mathrm{C}$ in silicone oil (Fig. S8, ESI $\dagger$ ).

Due to a weak adhesion between sequentially deposited layers, the mechanical properties of (layer-by-layer) additive manufactured materials are typically inferior to those of the corresponding bulk materials. In order to investigate the effect of the printing process and direction on the hydrogel's mechanical properties, we printed a solid rectangular stripe and then chemically cured for mechanical testing. In one g-code, the printing direction was chosen to be along the uniaxial stretching direction, in the other, the printing direction was perpendicular to it (Fig. 6c). As a control, a specimen cut from a molded bulk material was used. Remarkably, no significant influence of the printing process on the mechanical properties of hydrogels was observed (Fig. 6d). In fact, the Young's modulus (parallel $11.43 \pm 1.06 \mathrm{kPa}$, perpendicular $10.22 \pm$ $0.93 \mathrm{kPa}, n=4)$ are essentially identical to the one measured for bulk samples of $11.51 \pm 1.22 \mathrm{kPa}(n=4)$. Moreover, the printing process has no significant influence on the ultimate elongation, which remained at over 550\% elongation at break. Interestingly, samples printed in perpendicular direction exhibited a significantly higher toughness (a fracture strength of $35 \mathrm{kPa}$ )

(a)
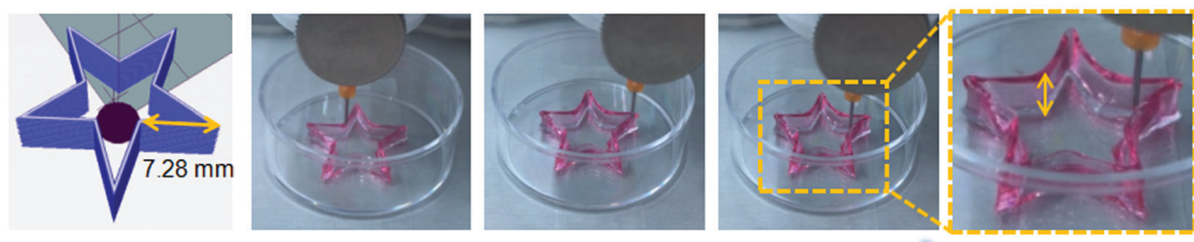

printing time

(b)
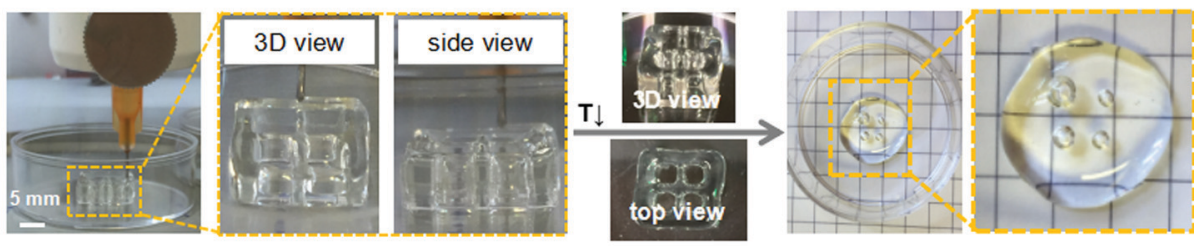

(c)
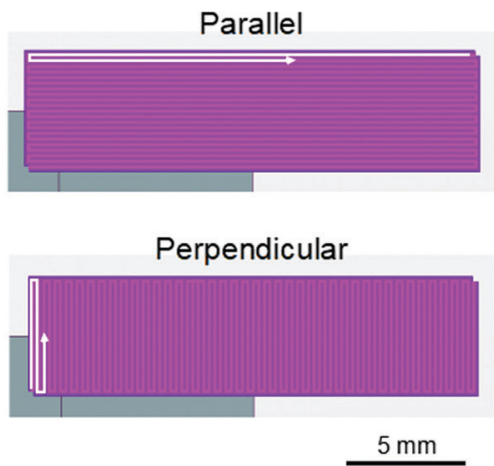

(d)

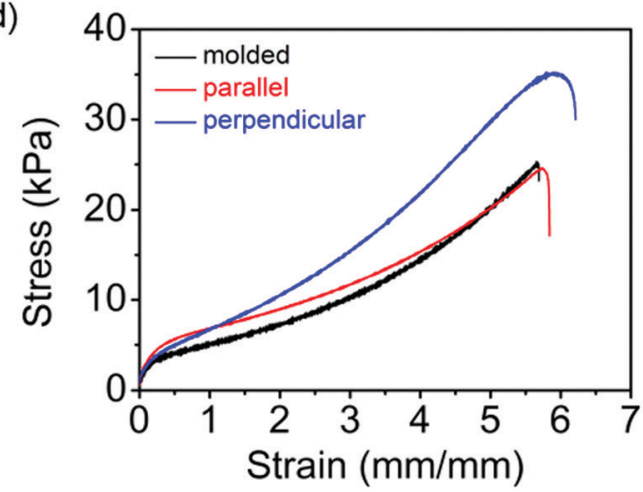

Fig. 6 (a) Screen shots of design and images showing the printing process of a 20-layers hollow five-pointed star $(7.28 \times 7.28 \times 5 \mathrm{~mm})$ with the hydrogel ink (dyed red by carmine). (b) 3D printing of a 20-layer cube $(3 \times 3$ lines) with the hydrogel ink. The printed cube collapsed gradually when the surrounding temperature drops below the $T_{\text {gel }}$. (c) Designed model of the hydrogel stripes printed with parallel or perpendicular filament orientation. (d) The stress-strain curves of hydrogel stripes printed parallel and perpendicular to the uniaxial stretching direction, and compared to molded bulk material. 
compared to the molded and parallel-printed one (both approx. $25 \mathrm{kPa}$ ). The fact that the layer-by-layer manufactured materials do not suffer with respect to their mechanical properties is only surprising on first sight and when thinking about layer-by-layer manufacturing of polymer melts. When extruding a line of polymer melt onto a solidified line, the macromolecular mobility and thus, entanglement will be severely hampered by the fact that one polymer strand is already solid and the other solidifies rapidly. Here, we print shear-thinning hydrogels, which are much softer viscoelastic solids that actually lack a primary phase boundary. Therefore, they are prone to mix and fuse on a molecular level much more effectively. Moreover, in the subsequent curing step, we polymerize a low molar mass monomer, which leads to the formation of an interpenetrating network, which naturally spans the hypothetical (from the g-code) layer boundaries. However, this does not explain why the part printed perpendicular shows a higher toughness. In fact, we made another unexpected observation. The hydrogel stripe printed in perpendicular to its long axis extended along the long axis while the stripe printed in parallel to its long axis extended perpendicular to it (Fig. S9, ESI $\dagger$ ). While the g-code for the print should in theory yield the same structure, it actually does not when the same printing parameters (extrusion pressure, needle speed) are used. This is likely related to the many turns in the perpendicular print, where the printhead must decelerate and accelerate again. We hypothesize that the slightly higher toughness is likely related to a discrepancy between the theoretical g-code and the actual extruded/printed structure. Such discrepancy has been recently discussed in a groundbreaking paper by Dalton and co-workers for melt electrowriting but we are not aware of such study in the context if direct ink writing. ${ }^{42}$ We propose that such study would be of great interest to the community.

In order to further investigate the printing behavior of the POx- $b$-POzi/DMAA/clay physical hydrogel and its performance after chemical curing, we designed and printed 3D constructs in various shapes. Here, the argon-purged silicone oil was used as a protective bath to avoid dehydration and the oxygen-induced (a) designed model

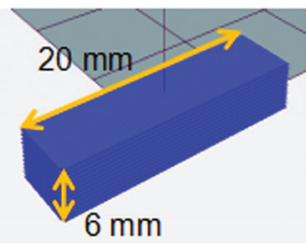

during printing

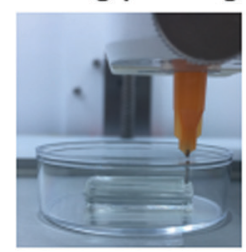

after polymerizing
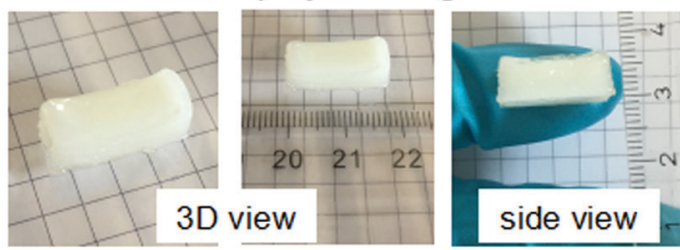

(b)

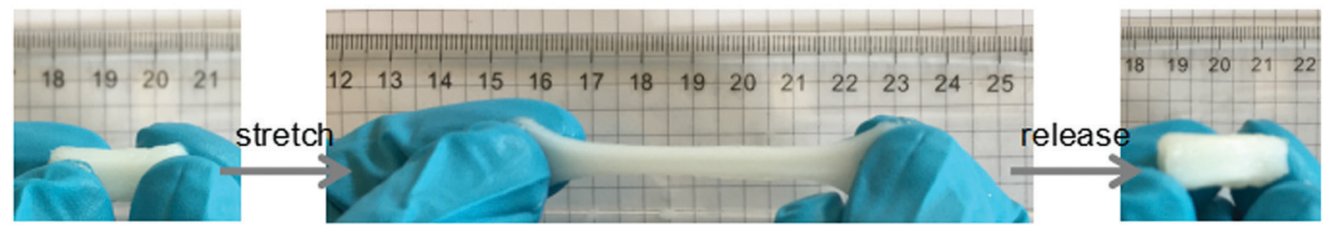

(c)
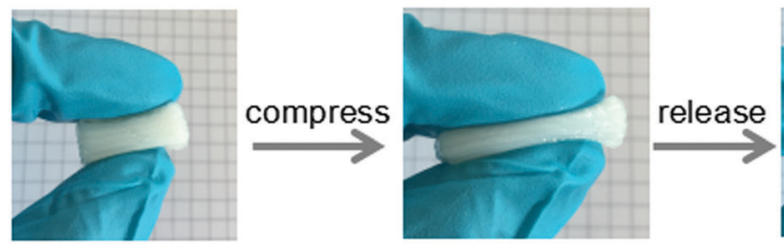

(e)
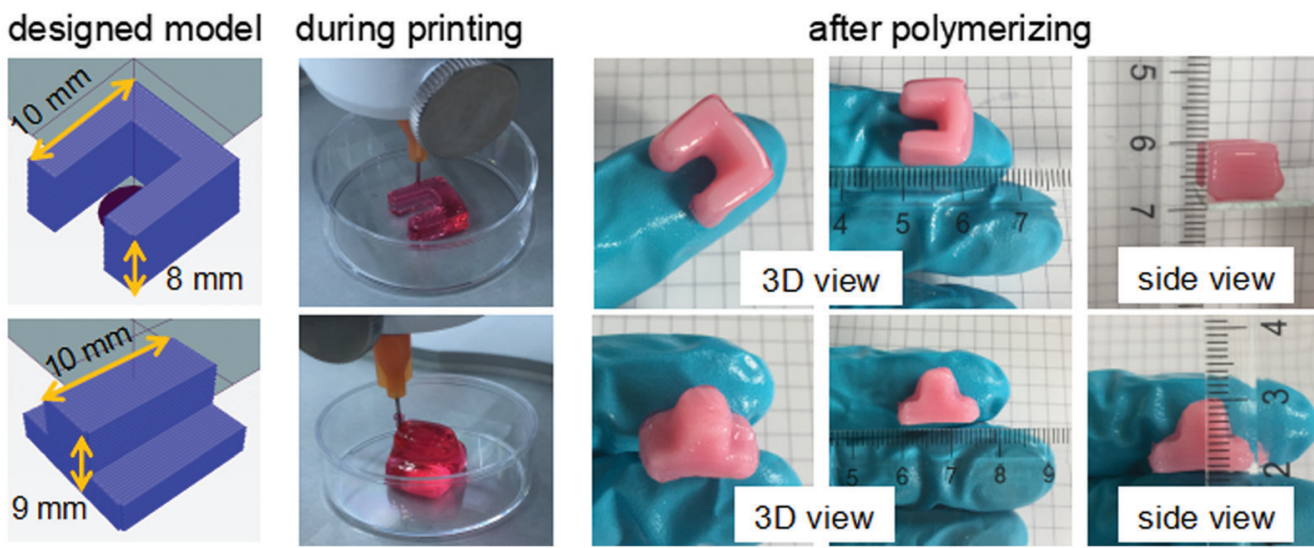

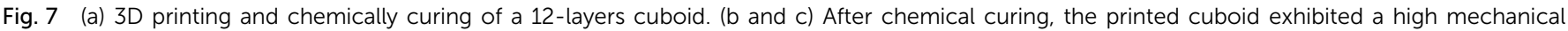

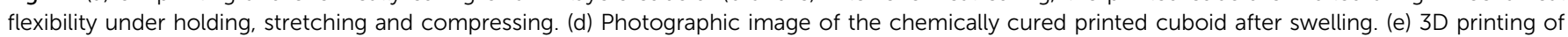
various constructs, and the corresponding printed results after chemical curing (dyed red by carmine). The photo background is $5 \times 5$ mm grid. 
inhibition of the free radical polymerization of DMAA. Initially, a 12-layers cuboid $(20 \times 5 \times 6 \mathrm{~mm})$ was printed under an extrusion pressure of $190 \mathrm{kPa}$, and then cured by incubating the printed structure at $60{ }^{\circ} \mathrm{C}$ overnight to initiate the polymerization of DMAA (Fig. 7a). The chemically crosslinked POx-b-POzi/PDMAA/ clay hydrogel cuboid was obtained successfully with a size of around $18 \times 5 \times 6 \mathrm{~mm}$. This suggest some shrinkage, which can be attributed to loss of water despite the silicone oil and shrinkage due to the radical polymerization. The cuboid exhibited remarkable flexibility upon stretching and compression (Fig. 7b and c), which is in good agreement with the mechanical tests discussed above. After equilibrium swelling in water, the printed POx- $b$ POzi/PDMAA/clay hydrogel cuboid increased in volume over 6-fold $(31 \times 10 \times 11 \mathrm{~mm})$ (Fig. 7d). Additionally, a 16-layer "U-shape" structure $(10 \times 10 \times 8 \mathrm{~mm})$ and an 18-layer "T-shape" structure $(10 \times 10 \times 9 \mathrm{~mm})$ were printed under an extrusion pressure of 190 $\mathrm{kPa}$, and were obtained successfully with good shape fidelity (Fig. 7e). In general, the thermoresponsive POx- $b$-POzi copolymer lays the foundation for the excellent printability of the POx- $b$-POzi/ DMAA/clay hybrid system, while the DMAA allows post-printing curing via free radical polymerization resulting in excellent mechanical strength with high stretchability.

\section{Conclusions}

In summary, a 3D printable POx-based POx- $b$-POzi/DMAA/clay ternary hybrid hydrogel was developed in this study, which can be further chemically cured by free radical polymerization. The resulting POx- $b$-POzi/PDMAA/clay hydrogels represent a clay reinforced IPN with good mechanical properties and high stretchability. Good 3D printability with high stackability and shape fidelity was demonstrated for various structures. The chemical crosslinking eliminates the original thermoresponsive property of the POx- $b$-POzi based hydrogel. The clay improves both, printability and mechanical properties of the final hybrid IPN hydrogel, as it significantly impacts the elongation at break, imparts excellent flexibility and compressability and facilitates handling. The novel hybrid IPN hydrogel presented here expands the material toolbox available for hydrogel-based 3D printing, and may be interesting for a wide range of applications including tissue engineering, drug delivery, soft robotics and additive manufacturing in general. Additionally, it is also expected to further promote the application of POx-based materials in the field of hydrogels.

\section{Conflicts of interest}

$\mathrm{RL}$ is listed as inventor on a patent pertinent to some materials in the present work. The authors declare no other competing financial interest.

\section{Acknowledgements}

This work was supported by the China Scholarship Council (CSC) and University of Würzburg. The authors would like to gratefully acknowledge support by the Deutsche Forschungsgemeinschaft (DFG, German Research Foundation) - project number 326998133 - TRR 225 (subproject A03), awarded to R. L. The authors gratefully acknowledge Isabell Biermann for her technical support with mechanical properties tests, and Ruben G. Scheuring for his technical support with the printer. Malik Salman Haider is grateful to higher education commission of Pakistan and German academic exchange services (HEC-DAAD Pakistan) for the award of PhD scholarship.

\section{References}

1 R. Luxenhofer, A. Schulz, C. Roques, S. Li, T. K. Bronich, E. V. Batrakova, R. Jordan and A. V. Kabanov, Biomaterials, 2010, 31, 4972-4979.

2 R. Luxenhofer, Y. Han, A. Schulz, J. Tong, Z. He, A. V. Kabanov and R. Jordan, Macromol. Rapid Commun., 2012, 33, 1613-1631.

3 N. Zhang, R. Luxenhofer and R. Jordan, Macromol. Chem. Phys., 2012, 213, 1963-1969.

4 A. Zahoranova, M. Mrlik, K. Tomanova, J. Kronek and R. Luxenhofer, Macromol. Chem. Phys., 2017, 218, 1700031.

5 T. Lorson, M. M. Lübtow, E. Wegener, M. S. Haider, S. Borova, D. Nahm, R. Jordan, M. Sokolski-Papkov, A. V. Kabanov and R. Luxenhofer, Biomaterials, 2018, 178, 204-280.

6 L. Hahn, M. M. Lübtow, T. Lorson, F. Schmitt, A. AppeltMenzel, R. Schobert and R. Luxenhofer, Biomacromolecules, 2018, 19, 3119-3128.

7 B. D. Monnery, V. V. Jerca, O. Sedlacek, B. Verbraeken, R. Cavill and R. Hoogenboom, Angew. Chem., Int. Ed., 2018, 57, 15400-15404.

8 R. Luxenhofer and R. Jordan, Mater. Matters, 2016, 8, 70.

9 Y. Chen, B. Pidhatika, T. von Erlach, R. Konradi, M. Textor, H. Hall and T. Lühmann, Biointerphases, 2014, 9, 031003.

10 T. R. Dargaville, J. R. Park and R. Hoogenboom, Macromol. Biosci., 2018, 18, 1800070.

11 A. Schwab, R. Levato, M. D’Este, S. Piluso, D. Eglin and J. Malda, Chem. Rev., 2020, 120, 11028-11055.

12 R. Levato, T. Jüngst, R. G. Scheuring, T. Blunk, J. Groll and J. Malda, Adv. Mater., 2020, 32, 1906423.

13 M. Hartlieb, K. Kempe and U. S. Schubert, J. Mater. Chem. B, 2015, 3, 526-538.

14 A. L. Fisher, J. M. H. Schollick, D. G. A. L. Aarts and M. C. Grossel, $R S C$ Adv., 2016, 6, 66438-66443.

15 F. A. Jerca, A. M. Anghelache, E. Ghibu, S. Cecoltan, I. C. Stancu, R. Trusca, E. Vasile, M. Teodorescu, D. M. Vuluga, R. Hoogenboom and V. V. Jerca, Chem. Mater., 2018, 30, 7938-7949.

16 L. Trachsel, N. Broguiere, J. G. Rosenboom, M. ZenobiWong and E. M. Benetti, J. Mater. Chem. B, 2018, 6, 7568-7572.

17 A. Zahoranova, Z. Kronekova, M. Zahoran, D. Chorvat, I. Janigova and J. Kronek, J. Polym. Sci., Part A: Polym. Chem., 2016, 54, 1548-1559. 
18 T. R. Dargaville, R. Forster, B. L. Farrugia, K. Kempe, L. Voorhaar, U. S. Schubert and R. Hoogenboom, Macromol. Rapid Commun., 2012, 33, 1695-1700.

19 J. Leppiniemi, P. Lahtinen, A. Paajanen, R. Mahlberg, S. Metsa-Kortelainen, T. Pinomaa, H. Pajari, I. VikholmLundin, P. Pursula and V. P. Hytonen, ACS Appl. Mater. Interfaces, 2017, 9, 21959-21970.

20 T. Jüngst, W. Smolan, K. Schacht, T. Scheibel and J. Groll, Chem. Rev., 2016, 116, 1496-1539.

21 Y. Huang, M. C. Leu, J. Mazumder and A. Donmez, J. Manuf. Sci. Eng., 2015, 137, 014001.

22 J. K. Placone and A. J. Engler, Adv. Healthcare Mater., 2018, 7, 1701161.

23 I. T. Ozbolat and M. Hospodiuk, Biomaterials, 2016, 76, 321-343.

24 S. V. Murphy and A. Atala, Nat. Biotechnol., 2014, 32, 773-785.

25 C. Mandrycky, Z. J. Wang, K. Kim and D. H. Kim, Biotechnol. Adv., 2016, 34, 422-434.

26 B. D. Monnery and R. Hoogenboom, Polym. Chem., 2019, 10, 3480-3487.

27 T. Lorson, S. Jaksch, M. M. Lübtow, T. Jüngst, J. Groll, T. Lühmann and R. Luxenhofer, Biomacromolecules, 2017, 18, 2161-2171.

28 M. M. Lübtow, M. Mrlik, L. Hahn, A. Altmann, M. Beudert, T. Lühmann and R. Luxenhofer, J. Funct. Biomater., 2019, 10, 36.

29 L. Hahn, M. Maier, P. Stahlhut, M. Beudert, V. Flegler, S. Forster, A. Altmann, F. Toppke, K. Fischer, S. Seiffert,
B. Bottcher, T. Lühmann and R. Luxenhofer, ACS Appl. Mater. Interfaces, 2020, 12, 12445-12456.

30 L. Trachsel, C. Johnbosco, T. Lang, E. M. Benetti and M. Zenobi-Wong, Biomacromolecules, 2019, 20, 4502-4511.

31 N. Paxton, W. Smolan, T. Böck, F. Melchels, J. Groll and T. Jüngst, Biofabrication, 2017, 9, 044107.

32 S. A. Wilson, L. M. Cross, C. W. Peak and A. K. Gaharwar, ACS Appl. Mater. Interfaces, 2017, 9, 43449-43458.

33 C. Hu, L. Hahn, M. Yang, A. Altmann, P. Stahlhut, J. Groll and R. Luxenhofer, J. Mater. Sci., 2020, 56, 691-705.

34 J. Chen, J. Huang and Y. Hu, ACS Appl. Mater. Interfaces, 2021, 13, 12726-12734.

35 S. Lee, E. S. Sani, A. R. Spencer, Y. Guan, A. S. Weiss and N. Annabi, Adv. Mater., 2020, 32, 2003915.

36 S. M. Hong, D. Sycks, H. F. Chan, S. T. Lin, G. P. Lopez, F. Guilak, K. W. Leong and X. H. Zhao, Adv. Mater., 2015, 27, 4035-4040.

37 C. W. Chang, A. van Spreeuwel, C. Zhang and S. Varghese, Soft Matter, 2010, 6, 5157-5164.

38 D. Chimene, C. W. Peak, J. L. Gentry, J. K. Carrow, L. M. Cross, E. Mondragon, G. B. Cardoso, R. Kaunas and A. K. Gaharwar, ACS Appl. Mater. Interfaces, 2018, 10, 9957-9968.

39 Y. F. Jin, C. C. Liu, W. X. Chai, A. Compaan and Y. Huang, ACS Appl. Mater. Interfaces, 2017, 9, 17457-17466.

40 S. Sinnwell and H. Ritter, Macromol. Rapid Commun., 2006, 27, 1335-1340.

41 S. Bennour and F. Louzri, Adv. Chem., 2014, 2014, 1-10.

42 A. Hrynevich, I. Liashenko and P. D. Dalton, Adv. Mater. Technol., 2020, 5, 2000772. 